\title{
The cartographic heritage of the University of Florence. Historical maps of the Geography Library
}

\author{
Margherita Azzari ${ }^{\text {a, } *}$, Camillo Berti ${ }^{\text {a }}$ \\ ${ }^{a}$ University of Florence, Department of History, Archaeology, Geography, Arts and Performing Arts, margherita.azzari@unifi.it, \\ camillo.berti@unifi.it \\ * Corresponding author
}

Keywords: Historical cartography, cultural heritage, academic library

\begin{abstract}
:
The Dipartimento di Storia, Archeologia, Geografia, Arte e Spettacolo (SAGAS) [Department of History, Archaeology, Geography, Arts and Performing arts] of the Università degli Studi di Firenze, houses the Geography Library in Fenzi Palace in via San Gallo, preserving a highly significant heritage consisting of volumes of geographical topics, historical maps, photographic collections and instruments.

This valuable collection was initially set up by the Chair of Geography of the Istituto di Studi superiori, pratici e di perfezionamento [Institute of Higher, Practical and Advanced Studies] (1859-1924), precursor of the present University, where some of the greatest masters of Italian geography worked, such as Giovanni and Olinto Marinelli, Attilio Mori, Renato Biasutti, Aldo Sestini, Giuseppe Barbieri (Cassi, 2016; Rombai, 2017). The university collections are attached to the collection of the Società di Studi Geografici [Society of Geographical Studies], founded in 1895, and the Marinelli Fund, which is the conspicuous collection of texts and papers created personally by Giovanni and Olinto Marinelli, owned by the Biblioteca Nazionale Centrale di Firenze, in perpetual loan to the University. These collections constitute a specialized documentary complex on geographical themes that for over a century has happily maintained a close connection - sharing its same premises - with the institutions that have enriched it since its establishment.

The map library, which is enhanced by a collection of ancient atlases and wall maps, has been further developed through progressive acquisitions and thanks to the union of various documentary bodies: the Marinelli Fund and the collections of the Institute of Geography of the Faculty of Letters, the Institute of Geography of the Faculty of Magisterium, and the Società di Studi Geografici. The historical section of the map library - which collects documents dating from the 1700s to the mid-1900s, even though the most conspicuous nucleus dates back to the period between the Unification of Italy and the First World War, includes a total of about 1,300 maps, many of which are divided into various sheets, for a total of almost 9,000 pieces. There are also several series (unfortunately not always complete) of the historical editions of the topographic maps of the Istituto Geografico Militare [Military Geographical Institute]; the most recent editions, in various scales (1: 25.000, 1: 50.000, 1: 100.000), are preserved in the modern section (Azzari et al., 2019).
\end{abstract}

The nearly 600 maps, for a total of 3,700 sheets, that are included in the Marinelli Fund, relate to a chronological range between the mid-18th century and the 1920s. This collection offers an insight into the geographic culture of the second half of the 19th century and of the early 20th century and of the cartographic production of this period: alongside editions in Italian, there are documents in Latin, German, French, English, Polish and many other languages, including Arabic and Chinese. The contents reflect the scientific, cultural and educational interests of Giovanni and Olinto Marinelli, also in the context of the network of local, national and international contacts maintained by the two Friulian geographers, as evidenced by the names of the authors (just to name a few: Guido Cora, Arcangelo Ghisleri, Giuseppe Della Vedova, Giovanni De Agostini, Giotto Dainelli, Stefano Sommier, Achille Dardano) and the publishers of the maps (among others: Touring Club Italiano, De Agostini, Istituto Geografico Militare, Justus Perthes). The maps, generally in a good state of conservation (even though some of them bear traces of the 1966 flood) are almost exclusively printed. However, some of them contain handwritten working notes by Giovanni and Olinto Marinelli or carry a dedication or the sender's name.

As for the geographical areas represented, in addition to a number of planispheres, this collection contains some sections relating to Asia and Oceania, the Americas and Africa, in which both small and medium-scale maps present the entire continent or some of its portions, states, territories and specific areas. Some sections are dedicated to Europe, where both comprehensive maps of the entire continent and general and thematic maps of individual states or regions are contained. Naturally, the largest collection of maps is dedicated to Italy, represented both as a whole and in its internal structure. In addition to some eighteenth-century examples, most of the preserved maps relate to cartographic production of the pre- 
unification states as well as those of the main national public and private post-unification cartographic institutes (Istituto Geografico Militare, De Agostini, Touring Club). There is a very wide range of typologies: from maps of individual regions or areas, including many city plans, to representations of particular themes of natural or anthropic forms, such as ports, lakes and volcanic forms. A rather large nucleus regards north-eastern Italy, and in particular the Friuli region, home of the Marinellis, and the province of Padua, where Giovanni held the chair of geography from 1878 until his transfer to Florence in 1893 (Azzari et al., 2019).

A notably rich collection of topographic maps is included in the cartographic fund of the former Institute of Geography: in addition to several series of the "Carta d'Italia" of the Istituto Geografico Militare in different scales, including the maps of the territories annexed at the end of the First World War, one can also find the complete topographic series of some European countries, such as France (in two editions), Switzerland, Germany, the Austro-Hungarian Empire. The collection of general and thematic maps of many European and non-European states is also particularly remarkable, and it includes valuable editions, such as the "Carte Générale du Royaume de la Nouvelle Espagne", created on the basis of Von Humboldt's observations in the early 1800s. Nevertheless, also in this section of the map library most of the documents are dedicated to Italy: alongside large-scale or chorographic maps, many of which are thematic, and numerous city plans, there are also historical editions of geological maps and the complete series of the "Carta Idrografica d'Italia" [Hydrographic Map of Italy] from the end of the 19th century.

The collection of "colonial" maps is especially worthy of mention. These derive from both the Marinelli Fund and the Geography Institute and Società di Studi geografici libraries, part of which was also probably inherited from the Florentine section of the African Society of Italy, founded in 1884 by Attilio Mori. It includes many maps regarding different parts of Africa of various origins, but the most significant nucleus is the Italian topographical production concerning the territories acquired or object of expansion interests by the Italian state: Eritrea and Ethiopia (1884-1941), Somalia (1890-1941), Libya (1913-1942). These are accompanied by maps of the Dodecanese isles (1912-1943) and Albania (1939-1943). The cartographic materials - approximately 400 maps in about 950 sheets - have different characteristics in terms of format, type and scale. In fact, alongside the actual maps, the collection hosts expedition reliefs and unpublished sketches (Azzari et al., 2011; Calloud, 2015).

With the dual aim of developing better conservation strategies and of promoting our cartographic (as well as photographic and documentary) heritage, an overall project has been launched with the goal of conducting a census, of cataloguing, and of conservative recovery. The project also includes digitization (using scanners for large format maps, and a planetary scanner and digital cameras for documents that cannot be scanned) and enabling open access for document web browsing. The ongoing activities aim to construct an information system with the goal of archiving and of the subsequent integrated

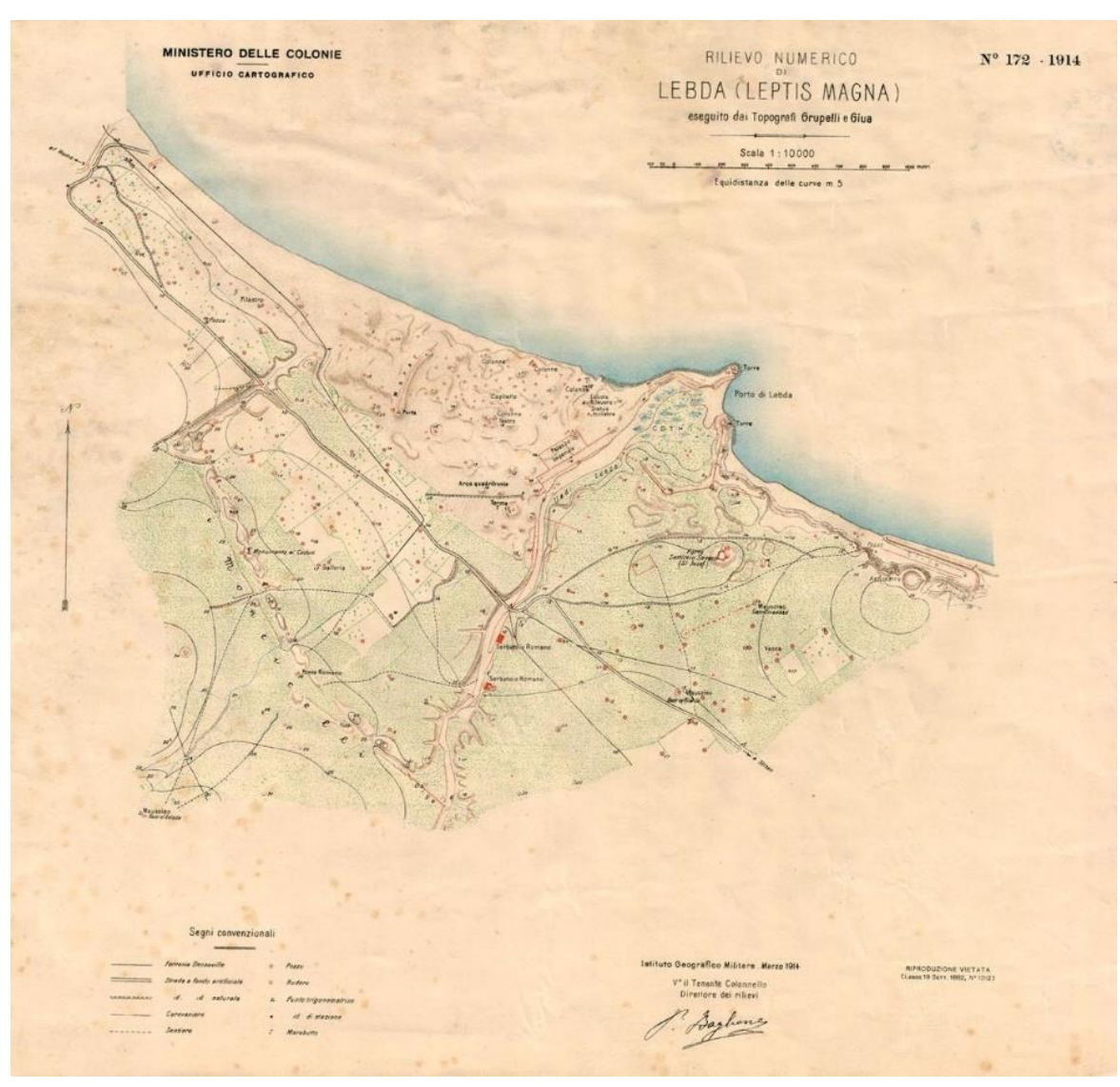
use of different types of documents: maps, photographs, diaries and handwritten notebooks, printed publications, working documents, sketches, etc. Overall, this library represents a cartographic patrimony of highly significant scientific and historical-cultural interest, little known even among specialists and hardly ever studied. Through its ongoing activities it is our intention to increase accessibility and usability for the public, in addition to improving the conservation and protection of this heritage.

Figure 1. One of the "colonial" maps in the historical map library: the "numerical relief" on a scale of 1:10,000 of the archaeological area of Leptis Magna in Libya (Ministero delle Colonie [Ministry of the Colonies], Firenze, Istituto Geografico Militare, 1914). 